\title{
Revisión de las tendencias teóricas e investigativas en el campo de la Psicología de la Actividad Física y del Deporte en Iberoamérica
}

\section{Review of theoretical and research trends in the field of Psychology of Physical Activity and Sports in Ibero-American}

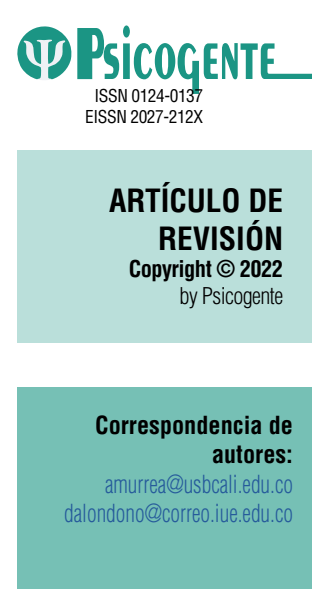

Recibido: $18-01-21$ Aceptado: 24-01-22 Publicado: 01-04-22

\author{
Ángela Urrea-Cuéllar iD \\ Universidad de San Buenaventura, Cali, Colombia \\ David Londoño Vásquez iD \\ Institución Universitaria de Envigado, Envigado, Colombia
}

Resumen

Introducción: Las tendencias teóricas e investigativas refieren propuestas y contemplaciones de los fenómenos sociales que se desarrollan en el campo de la psicología de la actividad física y del deporte, las cuales permiten tener otras perspectivas y formas de interactuar en el contexto.

Objetivo: Revisión de las tendencias teóricas e investigativas en el campo de la Psicología de la Actividad Física y del Deporte en Iberoamérica.

Método: La investigación fue cualitativa, hermenéutico-comprensiva. La unidad de análisis se conformó de 20 artículos de revistas iberoamericanas indexadas en Scopus en los años 2016 y 2017. Su selección se hizo a partir de los descriptores Deporte y Actividad Física. La búsqueda se realizó en el título, el resumen y las palabras clave. Para su análisis, se empleó una rúbrica, la cual permitió sistematizar la información bibliométrica del artículo, sus autores y textos citados y las palabras clave.

Resultados: Se identificaron los puntos de encuentro y las apuestas teóricas presentes en dichos artículos, agrupadas en trece categorías.

Conclusiones: Resulta significativa la presencia de categorías generales de la psicología como motivación, relacionamiento, habilidades para la vida e intereses de intervención dentro de aspectos tan disciplinarios como la Actividad Física y la Psicología del Deporte.

Palabras clave: psicología del deporte, actividad física, psicología del ejercicio, Iberoamérica, investigación, cualitativa, cuantitativa.

\section{Abstract}

Introduction: Theoretical and investigative tendencies refer to proposals and contemplations of the social phenomena that are developed in the field of psychology of physical activity and sport, which allow to have other perspectives and ways of interacting in the context.

Objective: Review of theoretical and research trends in the field of Psychology of Physical Activity and Sports in Ibero-American.

Method: The research was qualitative, hermeneutic-comprehensive. The analysis unit consisted of 20 articles of Ibero-American journals indexed in Scopus in 2016 and 2017. Their selection was made from two descriptors Sport and Physical Activity. The search was carried out in the title, the summary and the keywords. For its analysis, a rubric was used, which allowed systematizing the bibliometric information of the article, its authors and cited texts and the keywords.

Results: The agreement points and theoretical bets present in these articles were identified, grouped into thirteen categories.

Conclusions: It is significant the presence of general categories of psychology such as motivation, relationship, life skills and intervention interests in such disciplinary aspects as Physical Activity and Sports Psychology.

Keywords: sports psychology, physical activity, exercise psychology, Ibero-American, research, qualitative, quantitative.

Cómo citar este artículo (APA):

Urrea-Cuéllar, A. \& Londoño Vásquez, D. (2022). Revisión de las tendencias teóricas e investigativas en el campo de la Psicología de la Actividad Física y del Deporte en Iberoamérica. Psicogente 25(47), 1-23. https://doi.org/10.17081/psico.25.47.4836. 


\section{INTRODUCCIÓN}

Las Ciencias Sociales son un campo del conocimiento que se ha venido fortaleciendo y diversificando representativamente en los últimos 50 años (Wallerstein, 1996; De Sierra, 2007). El interés de los seres humanos por conocer los elementos que afectan o están presentes en las comunidades, la construcción de sociedades, las relaciones de poder, el individuo y el sujeto como actores de transformación, los fenómenos económicos y políticos que giran alrededor de una decisión, los aspectos éticos dentro de la interacción, los procesos de aprendizaje, el cómo se enseña algo, la construcción de noticias y la mejor forma para persuadir al otro, los comportamientos y las estructuras mentales, entre otros, han permitido que las Ciencias Sociales sean, cada vez, más representativas pero con unos límites más imprecisos de señalar. Estos intereses han permitido que las disciplinas que componen las Ciencias Sociales puedan estar en una tensión epistemológica frente a las Humanidades o Ciencias Humanas (Castro-Gómez, 2000; Archambault, Vignola-Gagné, Côté, Larivire \& Gingrasb, 2006).

A manera de ejemplo, la Organización para la Cooperación y Desarrollo Económico - OECD - (2018) propone que las disciplinas que componen las Ciencias Sociales son: ciencias políticas, derecho, economía, educación geografía, periodismo y comunicaciones, psicología y sociología. Por otro lado, Scimago clasifica las Ciencias Sociales en antropología, arqueología, bibliotecología, ciencia política, comunicación, demografía, desarrollo, derecho, educación, estudios culturales, de género y urbanos, geografía, lingüística y lenguaje, sociología y trabajo social (Bar-llan, 2008). En otras palabras, las agrupaciones de las disciplinas por campo del conocimiento responden a la concepción de las diferentes opciones epistemológicas. De igual forma, puede darse a la creciente interdisciplinariedad que se da en las investigaciones sociales y humanas (Arias, 2009).

Ahora es relevante señalar que la psicología es una disciplina que ha podido consolidarse, a la par de las Ciencias Sociales, especialmente, en Iberoamérica. En otras palabras, en estos cinco decenios, esta se ha diversificado en categorías como aplicada (Ford, MacCallum \& Tait, 1986; Moreno, 2013), clínica (Rotter, 1954; Reynoso \& Seligson, 2005), del desarrollo y educativa (Baltes, Reese \& Lipsitt, 1980; Gónzalez, Rodríguez e Imbert, 2004), experimental y cognitiva (Woodworth \& Schlosberg, 1954; Bravo, 2013), fisiológica (Kagan, Reznick \& Snidman, 1987; De la Fuente \& Leefmans, 2016), neuropsicológica (Shallice, 1988; Flores y Ostrosky, 2008) y social (Amabile, 1983; Sandoval, 2010). 
Dentro de la Psicología Aplicada, se encuentra la psicología de la actividad física y del deporte, la cual tiene su origen a principios del siglo XX (Wiggins, 1984), de donde se derivan cinco periodos de desarrollo de esta. El primero de ellos fue entre 1895-1920 en el cual se evidenciaron avances del campo en Norteamérica en el área de la investigación, específicamente con Triplett quien experimentó con ciclistas relacionando el área de psicología social y psicología del deporte (Weinberg y Gould, 2010); el segundo periodo (19211938) se caracterizó por la creación del primer laboratorio de psicología del deporte, a manos de Griffith a quien también se le considera como el padre de la psicología del deporte en América; en el siguiente periodo, entre 1939-1965, se produce el desarrollo científico del campo y se cuenta con el primer Congreso Mundial de Psicología, el cual se desarrolló en Roma en 1965 (Weinberg y Gould, 2010); entre 1966-1977, se considera que hubo un establecimiento académico del campo, por el desarrollo de cátedras en diversas universidades, las asesorías a equipos y deportistas, permitiendo con ello llevar al campo lo ejecutado en laboratorio; ya para el periodo comprendido entre 1978 y la actualidad se han realizado diversos procesos académicos y científicos entre ellos la creación de las revistas Journal of Sport Psychology (ahora conocida como Journal of Sport and Exercise Psychology), The Sport Psychologist, Journal of Applied Sport Psychology, International Journal of Sport Psychology y Psychology of Sport and Exercise; además, la Asociación de Psicología Americana (APA) reconoce la psicología del deporte en la división 47.

En Colombia se evidencia el desarrollo del campo a inicios de los años 70, donde aparecen temas de interés como el desarrollo psicomotor, el apoyo de Coldeportes para llevar a cabo "el primer evento académico sobre el área denominado Curso Suramericano de Psicología del Deporte" (Serrato, 2008, p.278), el incremento de la producción académica-científica y la creación de especializaciones, entre ellas, la Especialización en Psicología de la Actividad Física y del Deporte de la Institución Universitaria de Envigado, la cual sirve como marco institucional para la realización de este artículo.

Así mismo, Barbosa (2017) expresa que en las Universidades existe un mayor interés por parte de los estudiantes en este campo, así como de los deportistas, entrenadores y clubes por contar con el trabajo de dicho profesional, además de esto desde la empresa privada se muestra la importancia que se le da al campo, con la elaboración de lineamientos de políticas públicas en Psicología del Deporte (Coldeportes, 2016). 
Por su parte, Posada y Urrea (2018) encuestaron a los psicólogos del deporte que hay en Colombia, evidenciando que las áreas de intervención (que pueden ser más de una), donde el alto rendimiento es donde hay más presencia (72 psicólogos de 115 encuestados), seguido por los que se desempeñan en la docencia universitaria (46 psicólogos) y el área del ejercicio y la salud (31 psicólogos), esto deja entrever algunas de las tendencias que se han marcado en los últimos cinco años en la psicología del deporte, donde se ha reconocido el trabajo del psicólogo de esta área y el impacto que genera en las personas que están involucradas directa o indirectamente a la práctica de actividad física, ejercicio y deporte, esto reflejado en la presencia en Juegos Olímpicos y Paralímpicos, Juegos Suramericanos, Juegos Panamericanos y Parapanamericanos, etc. (MinDeporte, 2020).

\section{MÉTODO}

Dado al impacto que ha tenido la psicología de la actividad física y del deporte en Iberoamérica, se decidió realizar una investigación documental (Hoyos, 2000; Arias, Londoño, Gómez y Ortegón, 2015), cualitativa (Ñaupas, Mejía, Novoa y Villagómez, 2014; Denzin y Lincoln, 2015), hermenéutico-comprensiva (Herrera, 2009; Londoño-Vásquez, Olave-Astorga, Jaime-Salas y Losada-Salgado, 2018), que permitiera revisar las tendencias teóricas e investigativas presentes en las revistas de Psicología indexadas en Scopus en los años 2016 y 2017, en las categorías de Psicología Aplicada y Misceláneos a nivel de revistas iberoamericanas.

Se seleccionaron estas dos categorías teniendo en cuenta la relación que tiene la psicología del deporte y de la actividad física con la psicología aplicada (Weinberg y Goud, 2010; Moreno, 2013) y el potencial identificado en la categoría misceláneos (Arias, 2009; Tous y Mattar, 2012). Además, analizar el área Psicología, demandaría revisar 6 categorías más, las cuales no centran, necesariamente, su atención de publicación en estas temáticas (Bar-Ilan, 2008; Coldeportes, 2016).

Al cotejar la información disponible en Scopus para los años propuestos, frente a las dos categorías seleccionadas, se encontraron 11 revistas indexadas en psicología aplicada y 13 en misceláneos. De las 24 revistas identificadas, habían 6 en ambas categorías. Para un total de 18 revistas. Posteriormente, se procedió a analizar los diferentes números de dichas revistas en los años 2016 y 2017. 
Para este análisis, se utilizaron los descriptores Deporte y Actividad Física en el título, resumen y palabras clave de los artículos encontrados bajo las indicaciones enunciadas anteriormente. Se encontraron 38 artículos que cumplieran con los requisitos de selección: 2 en Q2, 16 en Q3 y 20 en Q4. Por tanto, para la unidad de análisis, de forma discrecional, se incluyeron el $100 \%$ de los artículos en Q2, el $50 \%$ de los de Q3 y Q4, para un total de 20 artículos. Los 18 correspondientes a Q3 y Q4, una vez se ubicaron en orden alfabético, se escogieron a través de la técnica de muestro conocida como aleatorio sistemático (Noy, 2008; Mendieta, 2015).

La unidad de análisis quedó conformada por los siguientes artículos:

Tabla 1.

Información de los artículos y revistas seleccionadas en la unidad de análisis

\begin{tabular}{|c|c|c|c|c|}
\hline NO. & NOMBRE DEL ARTÍCULO & REVISTA & AUTORES & CUARTIL \\
\hline 1. & $\begin{array}{l}\text { La promoción de valores a través de un pro- } \\
\text { grama de actividad física y predeportes en } \\
\text { personas con discapacidad intelectual leve }\end{array}$ & Siglo Cero (España) & $\begin{array}{c}\text { Elisa Isabel Sánchez-Romero, } \\
\text { M.a Pilar Vílchez Conesa y Cristina de } \\
\text { Francisco Palacios. }\end{array}$ & Q4/2017 \\
\hline 2. & $\begin{array}{l}\text { Validity evidence for the adaptation of the } \\
\text { State Mindfulness Scale for Physical Activity } \\
\text { (SMS-PA) in Spanish youth }\end{array}$ & Psicothema (España) & $\begin{array}{c}\text { Sarah Ullrich-French, Juan González y } \\
\text { María Dolores Hidalgo. }\end{array}$ & Q2/2017 \\
\hline 3. & $\begin{array}{c}\text { Unveiling anger and aggression in sports: } \\
\text { The effects of type of sport, competitive } \\
\text { category and success level }\end{array}$ & $\begin{array}{l}\text { Revista de Psicología del } \\
\text { Deporte } \\
\text { (España) }\end{array}$ & Rui Sofía y José Fernando Cruz & Q4/2017 \\
\hline 4. & $\begin{array}{c}\text { Motivación al deporte en adultos y personas } \\
\text { mayores que practican cachibol }\end{array}$ & $\begin{array}{l}\text { Cuadernos de Psicología del } \\
\text { Deporte } \\
\text { (España) }\end{array}$ & $\begin{array}{l}\text { Pedro Reynaga-Estrada, J. A. } \\
\text { García-Santana, } \\
\text { Edna Jáuregui-Ulloa, Cecilia Colun- } \\
\text { ga-Rodríguez, G. J. Carrera Viver y J. } \\
\text { L. Cabrera González. }\end{array}$ & Q3/2017 \\
\hline 5. & $\begin{array}{l}\text { Ejercicio físico agudo, agotamiento, calidad } \\
\text { del sueño, bienestar psicológico e intención } \\
\text { de práctica de actividad física }\end{array}$ & $\begin{array}{l}\text { Revista Iberoamericana de } \\
\text { Psicología del Ejercicio y el } \\
\text { Deporte } \\
\text { (España) }\end{array}$ & $\begin{array}{l}\text { Begoña Amador, Carlos Montero, } \\
\text { Vicente J. Beltrán-Carrillo, David } \\
\text { González-Cutre y Eduardo Cervelló }\end{array}$ & Q2/2017 \\
\hline 6. & $\begin{array}{c}\text { El papel del entrenador en la enseñanza de } \\
\text { competencias de vida a jóvenes brasileños } \\
\text { en riesgo de vulnerabilidad social }\end{array}$ & $\begin{array}{l}\text { Revista Universitas } \\
\text { Psychologica } \\
\text { (Colombia) }\end{array}$ & $\begin{array}{l}\text { Mauricio Marques, Catarina Sousa, } \\
\text { Jaume Cruz y Silvia Koller. }\end{array}$ & Q3/2016 \\
\hline 7. & $\begin{array}{c}\text { The effect of recording interval length on } \\
\text { behavioral assessment using the forced } \\
\text { swimming test }\end{array}$ & $\begin{array}{l}\text { Revista Iberoamericana de } \\
\text { Psicología y Salud } \\
\text { (España) }\end{array}$ & $\begin{array}{c}\text { Paloma Álvarez-Suárez, María Ban- } \\
\text { queri, Marina Vilella, Marta Méndez } \\
\text { y Jorge Arias. }\end{array}$ & Q4/2016 \\
\hline 8. & $\begin{array}{c}\text { Propiedades psicométricas de la versión } \\
\text { española del Cuestionario de Contenido de } \\
\text { Metas en el Ejercicio }\end{array}$ & $\begin{array}{l}\text { Revista Latinoamericana de } \\
\text { Psicología } \\
\text { (Colombia) }\end{array}$ & $\begin{array}{l}\text { Álvaro Sicilia, Manuel Alcaraz-Ibáñez, } \\
\text { María-Jesús Lirola y Rafael Burgueño. }\end{array}$ & Q3/2016 \\
\hline
\end{tabular}


Psychometric properties of the Brazi-

9. lian-adapted version of Sport Imagery Questionnaire

Percepción de los beneficios individuales de

10. uso de la bicicleta compartida como modo de transporte

Fatores motivacionais relacionados a prática de atividades físicas em idosos

Análisis psicométrico de la escala de

12. Autoconcepto AF5 de García y Musitu en estudiantes universitarios de Tarapoto (Perú)

Percepción de estrés y perfeccionismo en

13. estudiantes adolescentes. Influencias de la actividad física y el género

14.

Adaptación Española de la Escala de Pasión al Ámbito Deportivo

Apoyo a la autonomía, motivación, satisfac-

15. ción y niveles de actividad física en clases de educación física

16. Escala de Motivações para Jogos Online: Estudo de Adaptação à Realidade Brasileira

Impacto de la Entrevista Motivacional en la

17.

Adherencia de Pacientes Diabéticos Inactivos a la Actividad Física: Estudio Piloto de un Ensayo Clínico EMOACTIF- DM

Adaptación y validación al español del

18. cuestionario de habilidad en la práctica de la imaginación en el deporte.

Qualidade do Relacionamento Treinador-At-

19. leta e Orientação às Metas como Preditores de Desempenho Esportivo

20.

Dependência química e fortalecimento psicossocial pelas práticas esportivas
Psicologia: Reflexão

$$
\text { e Crítica }
$$

(Brasil)

Suma Psicológica

(Colombia)

Psicologia

em Estudo

(Brasil)

Interdisciplinaria: Revista de Psicología y Ciencias Afines (Argentina)

Ansiedad

y Estrés

(España)

Revista Iberoamericana de (Argentina) (Colombia)

Temas em Psicologia (Brasil)

Revista Colombiana de Psicología (Colombia)

Revista Mexicana de Psicología (México)

Psicologia: Teoria y Pesquisa (Brasil) Diagnóstico y Evaluación

Universitas Psychologica

Alberto Filgueiras y Craig Hall.

Q3/2017

Adriana Jakovcevic, Paul Franco,

Marcela Dalla y Rubén Ledesma.

Q3/2016

Agnes Navarro, Gabriela Kaiser, João Cren, Júlia Barreira y Paula Teixeira Fernandes.

Renzo Felipe Carranza y Milton Bermúdez-Jaimes.

Q3/2017

Antonio Muñoz y Juan González.

Ignacio Pedrosa, Eduardo GarcíaCueto, Julio Torrado y Constantino Arce

Gracielle Fin, Elisabeth Baretta, Juan Antonio Moreno-Murcia y Rudy José Nodari.

Valdiney Veloso Gouveia; Tailson Evangelista Mariano; Anderson Mesquita do Nascimento; Alex Sandro de Moura Grangeiro; Emerson Diógenes de Medeiros.

Ana María Muñoz-Flórez y

Olga Lucía Cortés-Ferreira.

Q4/2017

Manuel Alcaraz-Ibánez, José Manue Aguilar-Parra, Antonio Alías García y Ana Rodríguez Martínez.

Francielli Cheuczuk, Luciana Ferreira, Patric Flores, Lenamar Vieira, José Luiz Lopes y José Roberto Andare.

Q3/2016

Estudos de Psicologia (Brasil)
Marcelo Mendes.

Por otro lado, el análisis de los artículos seleccionados se realizó a través de la rúbrica propuesta por Ramírez (2020), la cual ya ha sido validada en dos investigaciones previas con propósitos metodológicos semejantes. El uso de este instrumento permitió consolidar el trabajo de análisis de los artículos seleccionados. Además, facilitó la consignación de los datos bibliográficos de 
dichos textos, los teóricos allí trabajados, los conceptos expuestos y las variaciones (transgresiones) dadas por el o los autor(es) del texto publicado.

Las categorías entre las que se agrupan las palabras claves de los artículos consultados son las siguientes: beneficios, calidad técnica en pruebas objetivas, competencia, cultura física, deportes, estados emocionales, estrategia, grupos poblacionales y géneros, habilidades para la vida, intereses de la intervención, investigación cuantitativa, motivación y relacionamiento.

\section{RESULTADOS}

\subsection{Relación entre cuartiles, años de publicación y nacionalidades de las} revistas

En total fueron 20 artículos los seleccionados como se mencionó en el apartado anterior. De estos, 2 en Q2, 8 en Q3 y 10 en Q4. Con respecto a las nacionalidades de las revistas, 7 artículos fueron publicados en revistas de España, 5 de Colombia, 5 de Brasil, 2 de Argentina y 1 de México. Además, estos artículos están distribuidos en los siguientes cuartiles y años:

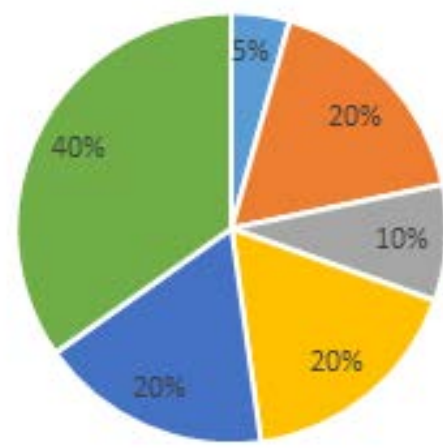

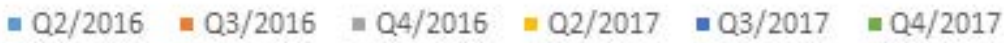

Figura 1. Relación de revistas por cuartiles y años

Este gráfico permite observar cómo la representación de los artículos de 2017 es significativamente superior en comparación con los de 2016, lo cual es recurrente con el corpus conformado por los 38 artículos.

De igual forma, si se analizan los artículos por cuartiles y nacionalidades de las revistas, se encuentran que España es el único país con revistas indexadas en Q2, dentro del corpus. Por otro lado, los artículos publicados en las revistas de Argentina, Brasil y Colombia representan el $87 \%$ de Q3. Con respecto a 
Q4, las revistas de Brasil, Colombia y España publicaron el 90 \%. Así como se señala en la siguiente gráfica.

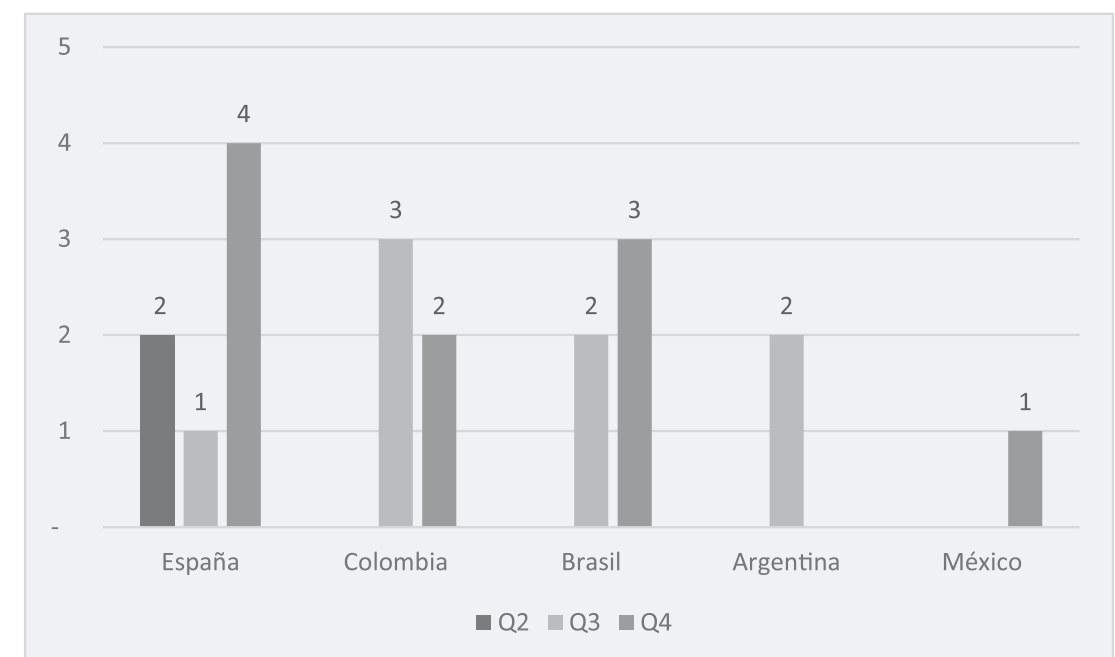

Figura 2. Relación de artículos discriminados por cuartiles y nacionalidades de las revistas

En cuanto a los autores, se identificaron 72 provenientes de 26 instituciones y 9 países. Estos están divididos así: 30 de España, 23 de Brasil, 5 de México, 4 de Argentina, 3 de Colombia, 2 de Ecuador, Estados Unidos y Portugal y 1 de Perú. Vale la pena mencionar que el promedio de autor por artículo es de 3,6.

No obstante, si se revisa esta información por cuartiles se obtiene que el promedio de autores por artículo Q2 y Q3 es de 4; mientras que, en Q4 es de 3,2 . Además, el mayor número de autores por artículo es de 6 , publicados en una revista española y otra brasileña, ambas indexadas en Q3.

\subsection{Autores y textos citados recurrentemente}

En los 20 artículos analizados, hay 190 autores citados más de 3 veces en los 76 textos referenciados. Es decir, un promedio de 2,5 autores por cada texto referenciado. Ellos son:

Tabla 2.

Relación autores citados en más de dos artículos del corpus

\begin{tabular}{|c|c|c|c|}
\hline AUTOR & $\begin{array}{l}\text { NO. ARTÍCULOS } \\
\text { CITADO }\end{array}$ & H INDEX & INSTITUCIÓN \\
\hline Allan Paivio & 2 & $\mathrm{~N} / \mathrm{A}$ & $\begin{array}{l}\text { Western Ontario University } \\
\text { (Canadá) }\end{array}$ \\
\hline Anthony Watt & 2 & 63 & $\begin{array}{c}\text { Victoria University } \\
\text { (Australia) }\end{array}$ \\
\hline
\end{tabular}




\begin{tabular}{|c|c|c|c|}
\hline \multicolumn{4}{|c|}{ Angela Urrea-Cuellar - David Londono Vasquez } \\
\hline Craig Hall & 2 & 63 & $\begin{array}{l}\text { Western Ontario University } \\
\text { (Canadá) }\end{array}$ \\
\hline vid González-Cutre & 2 & N/A & $\begin{array}{l}\text { Universidad Miguel Hernández } \\
\text { (España) }\end{array}$ \\
\hline Daniel Gould & 2 & 79 & $\begin{array}{c}\text { Michigan State University } \\
\text { (Estados Unidos) }\end{array}$ \\
\hline Diane Mack & 2 & 25 & $\begin{array}{c}\text { Brock University } \\
\text { (Canadá) }\end{array}$ \\
\hline Edward Deci & 4 & 144 & $\begin{array}{l}\text { University of Rochester } \\
\text { (Estados Unidos) }\end{array}$ \\
\hline eather Hausenblas & 2 & N/A & $\begin{array}{l}\text { Jacksonville University } \\
\text { (Estados Unidos) }\end{array}$ \\
\hline Richard Ryan & 4 & 172 & $\begin{array}{l}\text { Australian Catholic University } \\
\text { (Australia) }\end{array}$ \\
\hline Sophia Jowett & 2 & 55 & $\begin{array}{l}\text { Loughborough University } \\
\text { (Reino Unido) }\end{array}$ \\
\hline Tim Kasser & 2 & $\mathrm{~N} / \mathrm{A}$ & $\begin{array}{c}\text { Knox College } \\
\text { (Estados Unidos) }\end{array}$ \\
\hline William Black & 2 & 75 & $\begin{array}{l}\text { Colorado State University } \\
\text { (Estados Unidos) }\end{array}$ \\
\hline
\end{tabular}

Como se puede observar, un autor es de Reino Unido y otro de España, dos son de Australia, tres de Canadá y seis de Estados Unidos. Entre estos dos últimos países se concentra casi el $70 \%$ de los autores descritos. Por otro lado, cuatro de ellos no cuentan con $\mathrm{H}$ Index en Google Académico. Con respecto a aquellos autores con $\mathrm{H}$ Index, van desde 25 hasta 172.

De los autores de los artículos que componen el corpus, dos pertenecen al grupo de autores citados en dos artículos. Ellos son David González-Cutre y Craig Hall. El primero es coautor en la publicación Amador, Montero, BeltránCarrillo, González-Cutre y Cervelló (2017) y el segundo en Filgueiras y Hall (2017). Vale la pena mencionar que ambos autores referencian en dichos artículos, uno de sus textos publicados previamente.

Es importante mencionar que, de los 72 autores de los 20 artículos del corpus analizado, solo 2 son de Estados Unidos.

\subsection{Palabras clave}

Las palabras clave son, después del título y el autor, la opción de búsqueda más común en las consultas bibliográficas (Tous y Mattar, 2012). Gracias a la tecnología, las palabras clave han permitido generar rutas de trabajo asociadas a la revisión, agrupación y comparación de nominaciones o denominaciones alrededor de un concepto. Aspecto de interés, pues no siempre responde a una palabra precisa, sino a nominaciones compuestas, lo cual demuestra como complejizamos los conceptos en las diferentes áreas del conocimiento. 
Además, es necesario entender que tanto la citación como la referenciación, y en cierta medida las palabras clave, fungen como elemento de autoridad científica.

Teniendo en cuenta lo anterior, en este apartado, se agruparon las palabras clave de los 20 artículos, un total de 71 palabras (Ver Tabla 3). La apuesta, en esta ocasión, fue la de proponer categorías que agruparan dichas palabras clave. Estas categorías fueron validadas por expertos del campo de la Actividad Física y Psicología del Deporte ${ }^{1}$ :

Tabla 3.

Agrupación de palabras clave en categorías por expertos

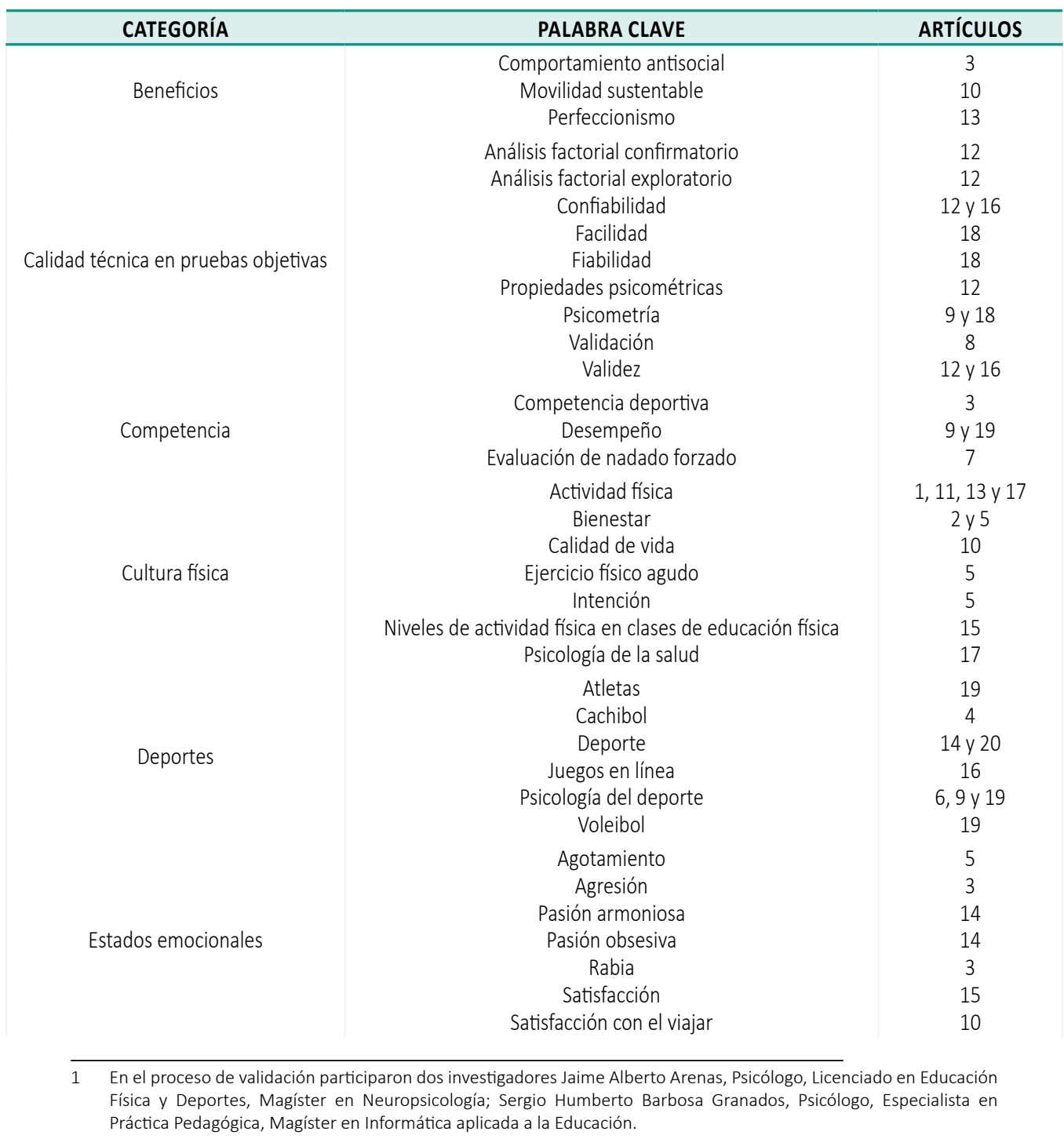




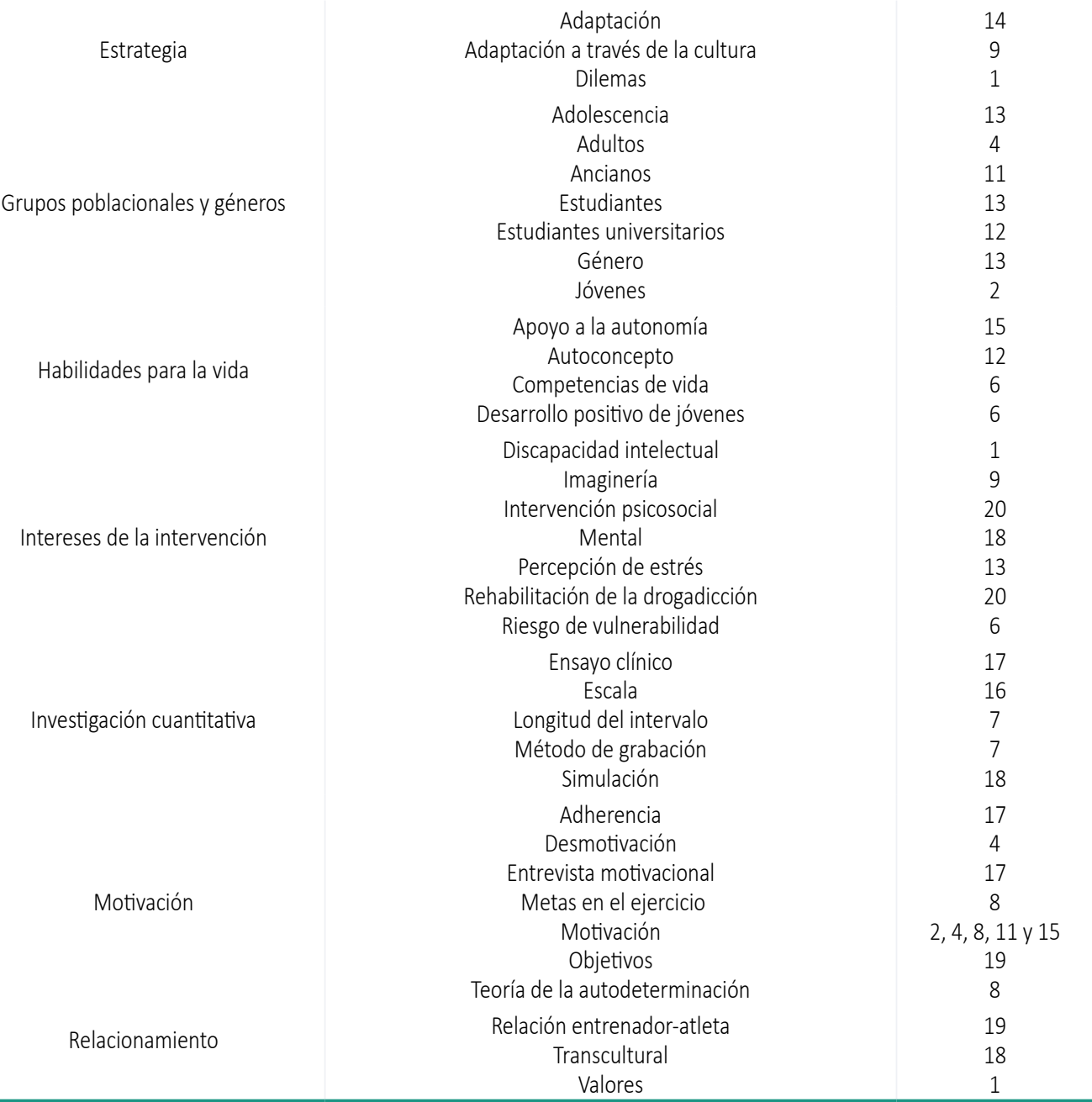

\section{DISCUSIONES}

Las categorías enunciadas anteriormente dejan entrever los diversos conceptos revisados por los autores. Esto permite realizar un análisis más detallado y así abordar las categorías más enunciadas en los artículos revisados. De allí, surgen los conceptos calidad técnica en pruebas objetivas, cultura física y motivación siendo las más citadas, y estados emocionales, grupos poblacionales y género, e interés de la intervención, los cuales dan una guía en este proceso de reconocimiento de las tendencias teóricas e investigativas. Por tanto, se analiza cada uno de estos conceptos con relación a los artículos analizados. Es necesario señalar que las trece categorías emanaron del análisis realizado a las palabras clave (Balestrini, 1987; Cázares, 1990). Por tanto, mantuvimos el orden en el cual identificamos tanto las categorías como las palabras que agrupamos: 
Beneficios: con respecto a esta categoría, las palabras clave que dieron soporte provienen de tres artículos. Uno de los artículos es el de Sofía y Cruz (2017), allí, abordan la relevancia de la ira y agresión en el deporte y sus efectos en el tipo de deporte, categoría competitiva y nivel de éxito; por su parte, Jakovcevic, Franco, Dalla Pozza y Ledesma (2016), analizan y dan cuenta sobre la percepción de los beneficios individuales del uso de la bicicleta compartida como modo de transporte; por último, Muñoz y González (2017) dan cuenta de las percepciones que tiene los estudiantes adolescentes sobre el estrés y las influencias de la actividad física y el género.

Calidad técnica en pruebas objetivas: esta categoría la componen nueve palabras claves, las cuales se reflejan en cinco artículos, estos son el de Carranza y Bermúdez-Jaimes (2017); Filgueiras y Hall (2017); Sicilia et al. (2017); Gouveia, Mariano, Nascimento, Grangeiro y Medeiros (2017); por último, Alcaraz-Ibáñez, Aguilar-Parra, Alías y Rodríguez (2017), dejan de manifiesto que la validación de cuestionarios o el análisis de los mismo, tienen un alto grado de validez y confiabilidad para la población en la cual se trabajó.

Competencia o competición: esta categoría la componen tres palabras claves, referenciadas en cuatro artículos. Sofia y Cruz (2017) retoman las variables psicológicas, ira y agresividad, y su influencia en la ejecución deportiva, mostrando que los deportes con más contacto físico y aquellos de categorías competitivas más bajas tienden a ser más agresivos. Por su parte, Álvarez-Suárez, Banqueri, Vilella, Méndez y Arias (2015) en su investigación utilizan la prueba de natación forzada, mostrando que hacen falta pruebas para hacer este tipo de registros, ya que ante estímulos aversivos los deportistas pueden tener diversas reacciones que afecten o no su desempeño. Filgueiras y Hall (2017) indican la imaginería en el deporte influyen en los procesos de competencia y el rendimiento. Por último, Muñoz-Flórez y Cortés-Ferreira (2017) expresan que la entrevista motivacional sirve para fortalecer la motivación intrínseca y evitar conductas de riesgo, como el sedentarismo.

Cultura física: esta categoría abarca siete palabras clave (actividad física bienestar, calidad de vida, ejercicio físico agudo, intención, niveles de actividad física en clases de educación física y psicología de la salud), que se describen en ocho artículos de la siguiente manera: Amador et al. (2017) concluyen que cada persona posee un nivel de tolerancia a la carga de entrenamiento, alcanzando niveles diferentes de agotamiento 
ante una misma sesión de ejercicio, lo que afectaría de modo diverso al bienestar psicológico. Muñoz-Flórez y Cortés-Ferreira (2017), por su parte, hallan en el estudio piloto "un incremento significativo en la actividad física realizada (caminada diaria) y una reducción en los niveles de glucosa en sangre en los pacientes intervenidos" (p.276). Otra de las investigaciones que refiere a la categoría es la de Sánchez-Romero et al. (2017), en la cual los valores involucrados en el desarrollo de la práctica deportiva, se establecen en las distintas situaciones y pueden guiar el comportamiento de las personas; Muñoz y González (2017) denotan que "El incremento de la práctica de ejercicio sin sentido deriva en una pérdida de control y la aparición de sentimientos negativos que aumentan la sensación de vulnerabilidad al estrés" (p.36); Jakovcevic et al. (2016) hacen un análisis más detallado, teniendo en cuenta la combinación de puntuaciones de importancia y efectos percibidos, los cuales satisfacen en mayor medida tres de los aspectos más valorados al momento de elegir un medio de transporte: la rapidez, el control del horario de llegada y el ahorro económico. Navarro, Kaiser, Cren, Barreira y Teixeira (2016) proponen que los factores que toman relevancia son salud, sociabilidad, control de estrés, estética y competitividad, que influyen en la práctica de actividad física en el anciano. Ullrich-French, González y Hidalgo (2017), en su investigación expresan que "Es importante aprender más sobre las experiencias de actividad física que pueden fomentar la motivación intrínseca y, a su vez, ayudar a fomentar una mayor participación y una salud óptima para los jóvenes españoles" ( $p$. 124); por último, la investigación que llevaron a cabo Fin et al. (2017), permitió señalar que "En contraste con el apoyo a la autonomía, es un estilo de control durante la clase. En este caso, los maestros se aseguran de que las actividades se realicen de acuerdo con su propia forma de pensar, sentir y comportarse" (p.3).

Deportes: se hace referencia de esta categoría en siete artículos donde se muestran seis palabras claves (atletas, cachibol, deporte, juegos en línea, psicología del deporte, voleibol) las cuales se involucran de diversas maneras, una de ellas, es la nueva tendencia de los juegos en línea y la motivación, la cual se evidencia desde tres aspectos realización, inmersión y el aspecto social (Gouveia et al., 2017); por su parte Pedrosa, García-Cueto, Torrado y Arce (2017), evidencian diferencias entre la pasión obsesiva y la armoniosa y sus implicaciones en la práctica de un deporte, además de proponer que la pasión armoniosa y el apoyo del entrenador hacia la autonomía son las variables externas al deportista. 
Mendes (2017) por su parte, presenta una propuesta de intervención que usa el deporte para combatir la dependencia del alcohol y otras drogas y el fortalecimiento psicosocial a través de la autoestima, la disciplina y la superación. Así mismo, Cheuczuk, Ferreira, Flores, Vieira, Lopes y Andare (2016), refiere que la orientación a la tarea y al ego de los deportistas, mostrando con ello, hacia dónde está orientado. Reynaga-Estrada, García-Santana, Jáuregui-Ulloa, Colunga-Rodríguez, Viver y CabreraGonzález (2017) ejecutan la investigación denominada Motivación al deporte en adultos y personas mayores que practican cachibol, donde lo relevante es la identificación de los motivos relacionados con el mantenimiento de la salud, la ocupación del tiempo libre, el encuentro con amigos y conocer gente. Lo que corresponde a esta categoría, se cierra con la investigación de Marques, Sousa, Cruz y Koller (2016) en la cual evalúan el papel del entrenador en la enseñanza de competencias de vida a jóvenes brasileños en riesgo de vulnerabilidad social, mostrando como a través de la práctica deportiva se puede persuadir el joven en dicha condición.

Estados emocionales: esta categoría conglomera siete palabras clave (agotamiento, agresión, pasión armoniosa, pasión obsesiva, rabia, satisfacción, satisfacción con el viajar) que se desarrollan en cinco textos consultados. Amador et al. (2017) expresan que a mayor agotamiento disminuye la vitalidad y se incrementaba el estado afectivo negativo, cada persona posee un nivel de tolerancia específico a la carga de entrenamiento, pudiendo percibir un nivel diferente de agotamiento ante una misma sesión de ejercicio; a su vez, Jakovcevic et al. (2016) exponen que es posible que los usuarios de mayor edad valoren más el efecto de la bicicleta sobre su control del horario de llegada ya que están sometidos a una mayor presión por cumplir con horarios y compromisos laborales que aquellos que son más jóvenes y no la usan para ir a trabajar; Pedrosa et al. (2017) ponen de manifiesto que "la pasión que experimenta el deportista afecta a las relaciones interpersonales intra y extradeportivas, entre las que se encuentra la relación con el entrenador" (p.172). Fin et al. (2017) muestran que la relación entre los factores sociales, en este caso el estilo interpersonal del maestro y el cumplimiento de las necesidades psicológicas básicas tiene efectos positivos a nivel cognitivo y afectivo; y Sofía y Cruz (2017) señalan que las variables ira y agresión en el deporte son las que han sido ampliamente estudiadas y se corrobora que ambas ocurren muchas veces con otras emociones durante el proceso competitivo, influenciándolo. 
Estrategia: esta categoría se compone de tres palabras clave, las cuales son adaptación, adaptación a través de la cultura y dilemas. SánchezRomero et al. (2017) ponen de manifiesto el tema de los valores, los cuales se pueden transformar o adaptar a ciertas circunstancias. Pedrosa et al. (2017) muestran las diferentes estrategias que pueden utilizar las personas que están vinculadas al medio con respecto a sus pasiones en este caso, las pasiones obsesivas y/o armoniosas; por último, Filgueiras y Hall (2016), también, manifiestan cómo la imaginería sirve como estrategia para el control en la competencia.

Grupos poblacionales y géneros: es una categoría que abarca siete palabras clave en cinco artículos. Carranza et al. (2017) abordan la población universitaria; Ullrich-French et al. (2017) hace adaptación de cuestionario en población joven; Reynaga-Estrada et al. (2017) retoman a los adultos y personas mayores; por su parte, Navarro et al. (2016) tienen como población de estudio a los ancianos con respecto a la variable motivación. Finalmente, Muñoz y González (2017) estudian la población adolescente con respecto al género y la percepción del estrés.

Habilidades para la vida: esta categoría está formada por cuatro palabras clave desarrolladas en tres artículos. Así, Marques et al. (2016), en la cual los autores desarrollan los términos de competencias de vida y desarrollo positivo de jóvenes mostrando cómo la figura del entrenador puede impactar positivamente en el riesgo de vulnerabilidad social; apoyo a la autonomía a través del desarrollo de habilidad en las clases de educación física (Fin et al., 2017) y el autoconcepto, revisado por Carranza et al. (2017) realizando el análisis psicométrico del AF5 en Perú.

Intereses de la intervención: es una categoría que amalgama siete palabras clave. En ellas, se denotan diferentes intereses. Uno de ellos es el trabajo con población con discapacidad intelectual donde SánchezRomero et al. (2017) revisan los valores a través de la práctica de actividad física. De igual forma, Filgueiras y Hall (2016) y Alcaraz-lbáñez et al. (2017) evidencian "la necesidad de intervenir de manera específica con el objeto de mejorar los procesos de imaginación en los que estén presentes imágenes alusivas al logro deportivo" (p.74). Otro de los procesos de intervención refiere al psicosocial, en el cual la actividad física o la práctica de un deporte sirve como promoción de la salud mental (Mendes, 2017); Muñoz y González (2017) dejan claro, que con respecto a la percepción del estrés se debe tener en cuenta la etapa del desarrollo de la persona, por los periodos de cambios (físicos, sociales 
y psicológicos). Por último, con respecto a la rehabilitación de la drogadicción y el riesgo de vulnerabilidad los entrenadores tienden a apoyar la labor educativa en este proceso (Marques et al., 2016).

Investigación cuantitativa: es una categoría que se compone de cinco palabras clave y se da cuenta en cuatro artículos, Álvarez-Suárez et al. (2015), Alcaraz-Ibáñez et al. (2017); Gouveia et al. (2017), y Muñoz-Flórez y Cortés-Ferreira (2017), fundamentan sus procesos en el método cuantitativo, es decir, que buscan cuantificar y aplicar una forma específica de análisis, que den cuente el objetivo del estudio.

Motivación: es una categoría que cuenta con siete palabras clave (adherencia, desmotivación, entrevista motivacional, metas en el ejercicio, motivación, objetivos, teoría de la autodeterminación). Esta es una de las categorías más estudiadas en lo que respecta a la psicología del deporte, desde sus diferentes teorías y una de las más utilizadas es la autodeterminación (Navarro et al., 2016; Ullrich-French et al.,2017; Sicilia et al., 2017; Fin et al., 2017; Reynaga-Estrada et al., 2017), otros artículos utilizan otras teorías entre ellas la de orientación a la meta (Cheuczuk et al., 2016 y Muñoz-Flórez et al., 2017)

Relacionamiento: esta última categoría agrupa tres palabras claves, las cuales son relación entrenador-atleta el cual se evidencia en el estudio realizado por Cheuczuk et al. (2016), transcultural (Alcaraz-lbáñez et al., 2017) y valores (Sánchez-Romero et al., 2017).

De acuerdo con los resultados enmarcados en esta investigación, se indica la importancia de las publicaciones en equipos de investigación para las revistas indexadas en Scopus. Dado a que solo 1 de los 20 artículos contó con un solo autor. Solo Western Ontario University aparece en dos ocasiones, pero corresponde a una misma publicación entre los autores Allan Paivio y Craig Hall, la cual fue en coautoría con Mack y Hasenblas en 1998. Por tanto, probablemente, este sea un texto de interés recurrente y reconocimiento en el campo de la Psicología del Deporte y la Actividad Física.

Por otro lado, los autores con más referencias en los artículos analizados son Edward Deci y Richard Ryan, cada uno con 4. Estos comparten tres publicaciones identificadas en el corpus (1985, 2000 y 2007). La publicación del 2000 está referenciada en los artículos de Fin, Baretta, Moreno-Murcia y Nodari (2017) y Sicilia, Alcaraz-Ibáñez, Lirola y Burgueño (2017). 
Esto permite inferir que el campo de la Psicología del Deporte y la Actividad Física, en España e Iberoamérica, si bien cuenta con avances investigativos y participación en la publicación de las revistas indexadas en Scopus, sigue teniendo como referencia conceptual y teórica los trabajos norteamericanos, en algunas ocasiones, concentrándose en la validación o replicación de los estudios realizados en estos países.

Lo que corresponde a esta agrupación de palabras clave en categorías, permite evidenciar tópicos que han marcado una tendencia en el área de la Psicología del deporte, como lo son: cultura física (Amador et al. , 2017), estados emocionales, motivación (Fin et al., 2017), relacionamiento (SánchezRomero, Vilchez Conesa y Francisco Palacios, 2017), habilidades para la vida, calidad técnica en pruebas objetivas (Filgueiras y Hall, 2017), investigación cuantitativa, además de ello, se muestran las nuevas propuestas como lo son: competencia (Sofía y Cruz, 2017), estrategia, interés de la intervención (Amador et al., 2017); las categorías deporte y grupos poblacionales y género dejan entrever las nuevas tendencias y la diversidad de población con la cual se ha trabajado.

\section{CONCLUSIONES}

Teniendo en cuenta lo anterior, se evidencia que para el campo de la Psicología de la Actividad Física y del Deporte, se ha radicado un interés significativo por la validación de escalas en diferentes países y poblaciones, mostrando con ello una necesidad específica frente a la calidad técnica y las posibilidades de generalización de los resultados.

Además, se identifica un mayor interés investigativo por la cultura física, donde caben los términos de actividad física, bienestar, calidad de vida, ejercicio físico agudo, intención, niveles de actividad física en clases de educación física y psicología de la salud.

Lo que corresponde a la categoría beneficios, se puede retomar lo expuesto por la OMS (2018)

los beneficios de la actividad física contrarrestan los posibles daños provocados, por ejemplo, por accidentes. Realizar algún tipo de actividad física es mejor que no realizar ninguna. Volviéndonos más activos a lo largo del día de formas relativamente simples podemos alcanzar fácilmente los niveles recomendados de actividad física (párr. 10). 
Lo anterior coincide con los artículos revisados y las palabras clave, llevando a concluir una vez más sobre el impacto en el cuerpo y la mente de la práctica de alguna actividad física o deporte.

De la misma manera, la competencia tal como lo enuncia Matveev (1983)

...el deporte en general, jamás se reduce al alcance de objetivos puramente competitivos, sino que es mucho más rico. Como actividad que influye de manera multifacética sobre el hombre... abarca la competición propiamente dicha, la preparación especial de la misma y la relación específica en el área de esta actividad tomadas en conjunto (pp.6-7).

Por ello, es que la categoría competencia se convierte o hace parte de la otra categoría motivación, que es una de las variables psicológicas más estudiadas en este campo, la cual presenta factores intrínsecos y extrínsecos, que van atados a los objetivos, a lo que se desee hacer y a lo que se pueda hacer, es decir, que también se involucra con las variables autoconfianza, autoconcepto, competencia, rendimiento, que se mueven con respecto a las necesidades de cada sujeto, hacia la tarea y el ego. Derivando los estados emocionales que son relevantes antes, durante y después de la práctica, ya que son estas, las que están presentes en la vida y, permiten que el ser humano se sienta vivo, porque las emociones son valores, que permiten la distinción entre las emociones negativas, positivas o algunas ambiguas, así como su momento de activación, dependiendo del momento que esté viviendo el ser humano, es decir, que influyen cuando un deportistas gana, se emociona y lo expresa saltando, sonriendo, brindando abrazos, etc.; mientras que cuando hay una pérdida, el deportista se retrae, evita a las personas, por ello, estas se consideran un medio para tener una zona de rendimiento óptimo (ZOF) (Hanin, 1978) adecuada, permitiendo el control de la ansiedad.

Por lo anterior, se concluye desde el corpus estudiado que existen diversos temas de interés en la psicología de la actividad física y del deporte que reflejan la actividad investigativa en este campo, teniendo en cuenta que algunos temas tienen mayor complejidad que otros, pasando a investigar temas genéricos como: la motivación, relacionamiento, habilidades para la vida, intereses de la intervención, a pasar a estudiar, temas específicos como deportes, grupos poblacionales y géneros, estados emocionales, la calidad de las pruebas, etc., lo cual ha permitido mayor apertura y visibilización del campo, creando nuevas teorías y tendencias que se evidenciaron 
en este proceso de revisión, incluyendo el uso del método científico desde las diferentes perspectivas de la investigación.

Lo anterior, permitirá enriquecer el campo a través de la generación de tendencias, teorías e investigativas, con respecto a las evidencias, teniendo en cuenta que el contexto es dinámico, al igual que las personas que están en él, las cuales tienen una mirada de la realidad diferente, la cual corresponde al rol que desempeñe.

Desde el grupo de investigación Pays, a través de la línea de Psicología Aplicada, se ha evidenciado la necesidad de hacer este tipo de estudios que permiten la consolidación de las especializaciones y la razón de ser de las mismas, especialmente, frente a la necesidad de ser coherentes con los desarrollos del campo y las tendencias investigativas que se pudieron identificar.

Conflicto de interés: Los autores declaran que no existe, de manera directa o indirecta, ningún tipo de conflicto de intereses, financieros, académicos ni personales para la publicación de este artículo de investigación.

Agradecimiento: A la Institución Universitaria de Envigado por todo el apoyo administrativo y académico para la realización de la presente investigación.

Financiamiento: Este trabajo fue financiado por la Oficina de Investigaciones de la IUE, bajo la modalidad de Iniciativa Propia, en el año 2019.

\section{REFERENCIAS}

Alcaraz-Ibáñez, M., Aguilar-Parra, J. M., Alías, A., y Rodríguez, A. (2017). Adaptación y validación al español del cuestionario de habilidad en la práctica de la imaginación en el deporte. Revista Mexicana de Psicología, 34(1), 65-76. https://www. redalyc.org/pdf/2430/243056045007.pdf

Álvarez-Suárez, P., Banqueri, M., Vilella, M., Méndez, M., \& Arias, J. L. (2015). The effect of recording interval length on behavioral assessment using the forced swimming test. Revista Iberoamericana de Psicología y Salud, 6(2), 90-95. https:// www.sciencedirect.com/science/article/pii/S217120691500006X

Amabile, T. M. (1983). The social psychology of creativity: A componential conceptualization. Journal of personality and social psychology, 45(2), 357. https://psycnet. apa.org/record/1984-06764-001

Amador, B., Montero, C., Beltrán-Carrillo, V. J., Gonzalez-Cutre, D., y Cervelló, E. (2017). Ejercicio físico agudo, agotamiento, calidad del sueño, bienestar psicológico e intención de práctica de actividad física. Revista iberoamericana de psicología del ejercicio y el deporte, 12(1), 121-127. https://dialnet.unirioja.es/servlet/ articulo?codigo $=5760452$

Archambault, É., Vignola-Gagné, É., Côté, G., Larivire, V., \& Gingrasb, Y. (2006). Benchmarking scientific output in the social sciences and humanities: The limits of existing databases. Scientometrics, 68(3), 329-342. https://akademiai.com/ doi/abs/10.1007/s11192-006-0115-z 
Arias, L. (2009). Interdisciplinariedad y triangulación en Ciencias Sociales. Diálogos Revista Electrónica de Historia, 10(1), 120-136. https://www.redalyc.org/ pdf/439/43913137005.pdf

Arias, O., Londoño, D., Gómez, D. y Ortegón, W. (2015). Campo científico de investigación en Negocios Internacionales. Facultad de Ciencias Empresariales, Institución Universitaria de Envigado. Envigado, Colombia: Institución Universitaria de Envigado.

Balestrini, M. (1987). Procedimientos Técnicos de la Investigación Documental. Caracas: Editorial Panapo.

Baltes, P. B., Reese, H. W., \& Lipsitt, L. P. (1980). Life-span developmental psychology. Annual review of psychology, 31(1), 65-110. https://www.annualreviews.org/doi/ abs/10.1146/annurev.ps.31.020180.000433?journalCode=psyc

Barbosa, S. (2017). Avances y retos de la psicología del deporte en el Eje Cafetero. En Retos y avances de la psicología del deporte en Colombia. Congreso Colombiano de Psicología: Psicología y Construcción de Paz. https://www.colpsic.org. co/aym_image/files/MEMORIAS\%20DEL\%20CONGRESO_Vs\%203_Dic\%2017.pdf

Bar-llan, J. (2008). Which h-index? A comparison of WoS, Scopus and Google Scholar. Scientometrics, 74(2), 257-271. https://link.springer.com/article/10.1007/ s11192-008-0216-y

Bravo, L. (2013). Lectura inicial y psicología cognitiva. Santiago de Chile: Ediciones UC.

Carranza, R. F., y Bermúdez-Jaimes, M. (2017). Análisis psicométrico de la Escala de Autoconcepto AF5 de García y Musitu en estudiantes universitarios de Tarapoto (Perú). Interdisciplinaria. Revista de Psicología y Ciencias Afines, 34(2). http:// www.ciipme-conicet.gov.ar/ojs/index.php/interdisciplinaria/article/view/350

Castro-Gómez, S. (2000). La reestructuración de las ciencias sociales en América Latina. Bogotá: Pensar, Instituto de Estudios Sociales y Culturales.

Cázares H., L. (1990). Técnicas actuales de investigación documental. (3ạ- Ed.) México: Trillas.

Cheuczuk, F., Ferreira, L., Flores, P. P., Vieira, L. F., Lopes, J. L., \& Andare, J. R. (2016). Qualidade do Relacionamento Treinador-Atleta e Orientação às Metas como preditores de Desempenho Esportivo. Psic.: Teor. e Pesq, 32(2), 01-08. http:// www.scielo.br/scielo.php?script=sci_arttext\&pid=S0102-37722016000200209

Coldeportes (2016). Lineamientos de Política Pública en Ciencias del Deporte. Bogotá: Coldeportes.

De la Fuente, R., y Leefmans, F. J. Á. (2016). Psicología médica. México: Fondo de Cultura Económica.

De Sierra, G. (2007). Las ciencias sociales en América Latina en perspectiva comparada. Buenos Aires: Siglo XXI Editores.

Deci, E. L \& Ryan, RM (1985). Intrinsic motivation and selfdetermination in human behavior. New York: Plenum.

Deci, E. L. \& Ryan, R. M. (2000). The what and why of goal pursuits: human needs and the self-determination of behavior. Psychological Inquiry, 11(4), 227-268. http:// dx.doi.org/10.1207/S15327965PLI110401

Denzin, N. y Lincoln, Y. (2015). Manual de Investigación Cualitativa - Métodos de recolección y análisis de datos. Barcelona, España: Gedisa Editorial.

Filgueiras, A., \& Hall, C. (2017). Psychometric properties of the Brazilian-adapted version of Sport Imagery Questionnaire. Psicologia: Reflexão e Crítica, 30(1), 22. https://prc.springeropen.com/articles/10.1186/s41155-017-0075-7 
Fin, G., Baretta, E., Moreno-Murcia, J. A., y Nodari, R. J. (2017). Apoyo a la autonomía, motivación, satisfacción y niveles de actividad física en clases de educación física. Universitas Psychologica, 16(4), 1-12. https://search.proquest.com/openview/ 9d869cfbafe3d207a4c1cd95580f736c/1?pq-origsite=gscholar\&cbl=2041157

Flores, J. C., y Ostrosky, F. (2008). Neuropsicología de lóbulos frontales, funciones ejecutivas y conducta humana. Revista neuropsicología, neuropsiquiatría y neurociencias, 8(1), 47-58.https://dialnet.unirioja.es/servlet/articulo?codigo=3987468

Ford, J. K., MacCallum, R. C., \& Tait, M. (1986). The application of exploratory factor analysis in applied psychology: A critical review and analysis. Personnel psychology, 39(2), 291-314. https://onlinelibrary.wiley.com/doi/ abs/10.1111/j.1744-6570.1986.tb00583.x

González, D. J., Rodríguez, M., e Imbert, N. (2004). Psicología educativa. La Habana: Editorial Pueblo y Educación.

Gouveia, V., Mariano, T., Nascimento, A., Grangeiro, A., \& Medeiros, E. (2017). Escala de motivações para jogos online: estudo de adaptação à realidade brasileira. Temas em Psicologia, 25(1), 131-141. https://dx.doi.org/10.9788/TP2017.1-08

Hall, C., Mack, D., Paivio, A., \& Hausenblas, H. (1998). Imagery use by athletes: Development of the Sport Imagery Questionnaire. International Journal of Sport Psychology, 29(1), 73-89. https://psycnet.apa.org/record/1998-04132-006

Hanin, Y.L. (1978). A study of anxiety in sports. Sport psychology: An analysis of athletic behavior. Ithaca, NY: Straub.

Herrera, J.D. (2009). La comprensión de lo social: horizonte hermenéutico de las ciencias sociales. Bogotá, Colombia: Cinde.

Hoyos, C. (2000). Un modelo para investigación documental. Guía teórico-práctica sobre construcción de Estados del Arte. Medellín, Colombia: Señal Editora.

Jakovcevic, A., Franco, P., Dalla Pozza, M. V., y Ledesma, R. (2016). Percepción de los beneficios individuales del uso de la bicicleta compartida como modo de transporte. Suma psicológica, 23(1), 33-41. https://www.sciencedirect.com/science/ article/pii/S0121438115000363

Kagan, J., Reznick, J. S., \& Snidman, N. (1987). The physiology and psychology of behavioral inhibition in children. Child development, 1459-1473. https://www. jstor.org/stable/1130685?seq=1\#metadata_info_tab_contents

Londoño-Vásquez, D. A., Olave-Astorga, J. M., Jaime-Salas, J. R. y Losada-Salgado, N. (2018). Lógicas, enfoques y epistemologías sobre educación y pedagogía. En Educación y Pedagogía trayectos recorridos (pp.96-154). Fondo Editorial Universidad de Manizales: Manizales.

Marques, M., Sousa, C., Cruz, J., y Koller, S. (2016). El papel del entrenador en la enseñanza de competencias de vida a jóvenes brasileños en riesgo de vulnerabilidad social. Universitas Psychologica, 15(1), 129-140. http://www.scielo.org.co/ scielo.php?pid=S1657-92672016000100010\&script=sci_abstract\&tlng=en

Matveev, L. (1983). Fundamentos del Entrenamiento Deportivo. Editorial Raduga, Moscú.

Mendes, M. (2017). Dependência química e fortalecimento psicossocial pelas práticas esportivas. Estudos de Psicologia, 22(3), 285-292. Disponible en: http://pepsic. bvsalud.org/scielo.php?script=sci_arttext\&pid=S1413-294X2017000300005

Mendieta, G. (2015). Informantes y muestreo en investigación cualitativa. Investigaciones Andina, 17(30), 1148-1150. Disponible en: https://www.redalyc.org/ pdf/2390/239035878001.pdf 
Ministerio del Deporte (2020). Consulta al Ministerio del Deporte por la participación de Psicólogos del deporte, a lo cual enviaron respuesta el 12 de marzo de 2020. Bogotá: MinDeportes.

Moreno, A. (2013). La psicología en el deporte. Revista deficiencias aplicadas al deporte, 5(12), 35-43. http://revistas.ut.edu.co/index.php/edufisica/article/ view/953/741

Muñoz, A., y González, J. (2017). Percepción de estrés y perfeccionismo en estudiantes adolescentes. Influencias de la actividad física y el género. Ansiedad y Estrés, 23(1), 32-37. https://www.sciencedirect.com/science/article/pii/S113479371730009X

Muñoz-Flórez, A., y Cortés-Ferreira, O. (2017). Impacto de la Entrevista Motivacional en la Adherencia de Pacientes Diabéticos Inactivos a la Actividad Física: Estudio Piloto de un Ensayo Clínico EMOACTIF-DM. Revista Colombiana de Psicología, 26(2), 263-281. https://www.redalyc.org/pdf/804/80454275006.pdf

Navarro, A., Kaiser, G., Cren, J., Barreira, J., \& Teixeira, P. (2016). Fatores Motivacionais Relacionados á Prática de Atividade Física em Idosos. Psicologia em Estudo, Maringá, 21(04), 677-685. https://www.redalyc.org/pdf/2871/287149565013. pdf

Noy, C. (2008). Sampling knowledge: The hermeneutics of snowball sampling in qualitative research. International Journal of social research methodology, 11(4), 327-344. https://www.tandfonline.com/doi/abs/10.1080/13645570701401305

Ñaupas, H., Mejía, E., Novoa, E. y Villagómez, A. (2014). Metodología de la investigación cuantitativa - cualitativa y redacción de la tesis. Bogotá, Colombia: Ediciones de la U.

OECD. (2018). Perspectivas económicas de América Latina 2018: Repensando las instituciones para el desarrollo. París: OECD Publishing.

OMS. (2018). Actividad Física. https://www.who.int/es/news-room/fact-sheets/ detail/physical-activity

Pedrosa, I., García-Cueto, E., Torrado, J., y Arce, C. (2017). Adaptación española de la Escala de pasión al ámbito deportivo. Revista Iberoamericana de Diagnóstico y Evaluación Psicológica, 1(43), 165-176. https://www.aidep.org/sites/default/ files/articles/R43/Art12.pdf

Posada, Z. y Urrea, A. (2018). Características del ejercicio profesional del psicólogo del deporte en Colombia. Revista COLREPD, 1, 1-12. https://colrepd.net/caracteristicas-del-ejercicio-profesional-del-psicologo-del-deporte-en-colombia/

Ramírez, A. (2020). Tendencias investigativas y referentes en los campos de saber: el caso de las líneas de investigación del grupo PAYS. Envigado: Fondo Editorial IUE.

Reynaga-Estrada, P., García-Santana, J. A., Jáuregui-Ulloa, E. E., Colunga-Rodríguez, C., Viver, G. C., y Cabrera-González, J. L. (2017). Motivación al deporte en adultos y personas mayores que practican cachibol. Cuadernos de Psicología del Deporte, 17(2), 15-26. https://revistas.um.es/cpd/article/view/301801

Reynoso, L., y Seligson, I. (2005). Psicología clínica de la salud: un enfoque conductual. México: UNAM.

Rotter, J. B. (1954). Social learning and clinical psychology. New Jersey: Prentice-Hall.

Ryan, R. M., \& Deci, E. L. (2007). Active human nature: Selfdetermination theory and the promotion and maintenance of sport, exercise, and health. In M. S. Hagger \& N. Chatzisarantis (Eds.), Intrinsic motivation and self-determination in exercise and sport (pp. 1-19). Champaign, IL: Human Kinetics.

Sánchez-Romero, E. I., Vílchez Conesa, M., y Francisco Palacios, C. (2017). La promoción de valores a través de un programa de actividad física y predeportes en personas 
con discapacidad intelectual leve. Siglo Cero: Revista Española sobre Discapacidad Intelectual, 48(3), 73-86. http://riberdis.cedd.net/handle/11181/5451

Sandoval, J. (2010). Construccionismo, conocimiento y realidad: una lectura crítica desde la Psicología Social. Revista Mad, (23), 31-37. https://revistaderechoambiental.uchile.cl/index.php/RMAD/article/view/13633

Serrato, L. H. (2008). Historia de la psicología del deporte en Colombia. Revista iberoamericana de psicología del ejercicio y el deporte, 3(2), 277-300. http:// www.redalyc.org/articulo.oa?id=3111/311126260009

Shallice, T. (1988). From neuropsychology to mental structure. Cambridge: Cambridge University Press.

Sicilia, Á., Alcaraz-Ibáñez, M., Lirola, M. J., y Burgueño, R. (2017). Propiedades psicométricas de la versión española del Cuestionario de Contenido de Metas en el Ejercicio. Revista Latinoamericana de Psicología, 49(3), 182-193. https://www. sciencedirect.com/science/article/pii/S0120053417300249

Sofia, R., \& Cruz, J. F. A. (2017). Unveiling anger and aggression in sports: the effects of type of Sport, competitive category and success level. Revista de psicología del deporte, 26(2), 21-28. https://www.redalyc.org/pdf/2351/235152048003.pdf

Torres, A., y Jiménez, A. (2004). La práctica investigativa en ciencias sociales. Bogotá: Universidad Pedagógica Nacional.

Tous, M. G. y Mattar, S. (2012). Las claves de las palabras clave en los artículos científicos. Revista MVZ Córdoba, 17(2), 2955-2956. https://doi.org/10.21897/ rmvz.228

Ullrich-French, S., González, J., \& Hidalgo, M. D. H. (2017). Validity evidence for the adaptation of the State Mindfulness Scale for Physical Activity (SMS-PA) in Spanish youth. Psicothema, 29(1), 119-125. http://psicothema.com/pdf/4373.pdf

Veloso, V., Evangelista, T., Mesquita, A., de Moura, A., \& Diégenes, E. (2017). Escala de motivações para jogos online: estudo de adaptação à realidade brasileira. Temas em Psicologia, 25(1), 131-141. https://www.redalyc.org/pdf/5137/Resumenes/ Resumen_513754916008_1.pdf

Wallerstein, I. (Ed.). (1996). Abrir las ciencias sociales: informe de la Comisión Gulbenkian para la reestructuración de las ciencias sociales. Buenos Aires: Siglo XXI Editores.

Weinberg, R. y Gould, D. (2010). Fundamentos de Psicología del Deporte y del Ejercicio Físico (4a. ed.). Bogotá, Colombia: Editorial Médica Panamericana.

Wiggins, D. K. (1984). The history of sport psychology in North America. En J. M. Silva y R. S. Weinberg (eds.), Psychological foundations of sport (pp. 9-22). Champaign, IL, Human Kinetics.

Woodworth, R. S., \& Schlosberg, H. (1954). Experimental psychology. Oxford: IBH Publishing. 